\title{
Urine biomarkers in renal allograft
}

\author{
Hongting Wang, ${ }^{1,2}$ Zuan-tao Lin ${ }^{1}$, Yulin Yuan ${ }^{1,3}$, Tianfu $\mathbf{W u}^{1}$ \\ ${ }^{1}$ Department of Biomedical Engineering, University of Houston, Texas, USA; \\ ${ }^{2}$ National Pharmacology Laboratory of Chinese Medicine, Basic Medical College. Wannan Medical College, \\ Wuhu 241002, Anhui Province, China; \\ ${ }^{3}$ Department of Clinical Laboratory, the People's Hospital of Guangxi Zhuang Autonomous Region, Nanning \\ 530021, Guangxi Zhuang Autonomous Region, China
}

\section{ABSTRACT}

There is a high risk for the survival of patients with an end-stage renal disease for kidney transplantation. To avoid rejection by strict medication adherence is of utmost importance to avoid the failure of a kidney transplant. It is imperative to develop non-invasive biomarkers to assess immunity risk, and to ultimately provide guidance for therapeutic decision-making following kidney transplantation. Urine biomarkers may represent the promising non-invasive tools that will help in predicting risk or success rates of kidney transplantations. Furthermore, composite urinary biomarkers or urinary biomarker panel array might be critical in improving the sensitivity and specificity in reflecting various risks of kidney failure during transplantation. This review primarily focuses on the role of such biomarkers in predicting chronic kidney disease (CKD) progression and/or cardiovascular disease (CVD) risk in renal allograft.

Key words: kidney transplantation, urine biomarkers, biomarker panel array

\section{INTRODUCTION}

Kidney transplantation is the organ transplant of a kidney into a patient with end-stage renal disease (ESRD). Renal allograft has significantly improved the survival of patients with ESRD. Renal allograft is typically classified as deceased-donor or living-donor transplantation depending on the source of the donor organ. ${ }^{[1]}$ According to the Organ Procurement and Transplant Network (OPTN) records, nearly 85,000 candidates were on the waiting list for kidney transplantation in 2010 , and $\approx 17,700$ kidney transplantations (including 828 kidneypancreas transplantations) were performed. [2] The data from the United States Renal Data System (USRDS) shows that currently approximately one in three donations is from a live donor. The other donations of organs are used clinically for renal allograft obtained from the deceased donors. Despite advances in medicine and technology, and increased awareness of organ donation and transplantation, the gap between supply and demand continues to widen. In the United States, 17,000 kidney transplantations were done in 2014, but at least five-fold more patients are on the waiting list, and each day 12 people die while waiting for a life-saving kidney transplant. ${ }^{[2]}$ Therefore, alternatives to human kidney transplants from living or deceased donors are thus urgently needed. In addition, the donor and recipient should be ABO blood group and cross match (HLA antigen) compatible. ${ }^{[3]}$ If a potential living donor is incompatible with the recipient, the donor could be exchanged for a compatible kidney. ${ }^{[4]}$ Kidney exchange, also known as "kidney paired donation" or "chains", has recently gained popularity, over the past few years. The reasons why kidneys fail over time after transplantations has been reported in recent years. On the other hand, transplant rejections still exist between supply and demand in the same $\mathrm{ABO}$ blood and compatible HLA antigen group. A transplant rejection occurs when the transplanted tissue is rejected by the recipient's immune system, which destroys the transplanted tissue. ${ }^{[5-7]}$ Avoiding rejection by strict medication adherence is of utmost importance to avoid failure of the kidney transplant. Therefore, effective monitoring for complications of kidney transplantation depends on frequent invasive or non-invasive procedures. 
Wang et al.: Urine biomarkers in renal allograft

A biomarker is defined as a "cellular, biochemical or molecular alteration in cells, tissues or fluids that can be measured and evaluated to indicate normal biological processes, pathogenic processes, or pharmacological responses to a therapeutic intervention. ${ }^{[8]}$ The serum creatinine is a poor marker of early renal dysfunction, because the serum concentration is greatly influenced by numerous non-renal factors (such as body weight, race, age, gender, total body volume, drugs, muscle metabolism, and protein intake). ${ }^{[9]}$ Urine biomarker plays a crucial role to diagnose transplant rejection that occurs when the transplanted tissue is rejected by the recipient's immune system.

Recent technological advances have resulted in the identification of a growing number of potential renal biomarkers in the urine of patients and animal models of kidney diseases. ${ }^{[10]}$ Many of these are still awaiting further testing and clinical validation. However, it is becoming clear that these renal biomarkers can be grouped into different categories. SG Coca et al. performed a systematic review of publications that evaluated the accuracy and reliability of the serum and urinary biomarkers in human subjects, when used for the diagnosis of established acute kidney injury (AKI) or early AKI, or to stratify risk of patients with AKI. ${ }^{[1]]}$ The results of their studies indicated that urine neutrophil gelatinase-associated lipocalin (NGAL), kidney injury molecule-1 (KIM-1), interleukin-18 (IL18), glutathione-S-transferase (GST), N-acetyl- $\beta$-Dglucosaminidase (NAG), alpha-1 microglobulin $(\alpha-1-\mathrm{M})$, matrix metalloproteinase-9 (MMP-9), N-acetyl- $\beta$-Dglucosaminidase (NAG) and sodium hydrogen exchanger 3 (NHE3). The performance of these biomarkers was the best for the differential diagnosis of established AKI. Published data from the studies of serum and urinary biomarkers suggested that these biomarkers may have great potential to advance the fields of kidney transplantation. Many researchers discovered and redefined a serial of cytokines to influence the rate of renal survival after kidney allograft. Kim et al. ${ }^{[12]}$ summarized that several urine biomarkers have been correlated with allograft injury, including NGAL, IL-18, cystatin C and KIM-1. So far, noninvasive biomarkers are still urgently needed to assess the immune risk, and ultimately to guide therapeutic decisionmaking following a kidney transplantation.

Herein we are summarizing the reported urinary biomarkers which might have great potential in predicating the risk of renal allograft.

\section{LIVER FATTY ACID BINDING PROTEIN}

The liver fatty acid binding proteins (LFABP) are a family of transport proteins for fatty acids and other lipophilic substances. The urinary liver-type fatty acid-binding protein is a proximal tubular injury candidate biomarker for early detection of acute kidney injury (AKI), with variable performance characteristics depending on the clinical settings. $\mathrm{LFABP}^{[13]}$ were identified as putative rejection biomarkers. Among the markers tested for diagnosing acute rejection (AR), urinary CXCL9 mRNA and CXCL9 protein were the most robust ${ }^{[14]}$ markers.

\section{CHEMOKINE LIGAND 9}

Chemokine ligand 9 (CXCL9) is a small mediator belonging to the CXC chemokine family that is also known as Monokine induced by gamma interferon (MIG). CXCL9 is a T-cell chemoattractant that is induced by IFN- $\gamma$. It is closely related to two other CXC chemokines called CXCL10 and CXCL11, whose genes are located near the gene for CXCL9 on human chromosome ${ }^{[15]}$. The mechanism study revealed that the T cell-attracting chemokines CXCL9 and CXCL10 induced by doxorubicin accounted for the recruitment of $\mathrm{T}$ cells to tumors, and the tumor-specific up-regulation of IFN $\gamma$, following IL-12 signaling initiate the positive feedback to stimulate $\mathrm{T}$ cell proliferation. The approach of IL-12 plus doxorubicin followed by murine $\mathrm{T}$ cell and human TIL infusion yielded striking response in murine models. ${ }^{[16]}$

\section{TISSUE INHIBITOR METALLOPROTEINASE-2 AND INSULIN-LIKE GROWTH FACTOR- BINDING PROTEIN 7}

Tissue inhibitor metalloproteinase-2 (TIMP-2) is a human gene, thought to be a metastasis suppressor. The insulinlike growth factor-binding protein 7 (IGFBP7) is a protein that is encoded by the IGFBP7 gene in humans. The major function of this protein is to regulate the availability of insulin-like growth factors (IGFs) in tissue as well as in modulating IGF binding to its receptors. The combination of urinary TIMP-2 and IGFBP7 serves as a sensitive and specific biomarker to predict AKI early after cardiac surgery and to predict renal recovery ${ }^{[17]}$. Urinary TIMP-2 and IGFBP7 were identified as patients at risk for imminent $\mathrm{AKI}^{[18]}$.

\section{CHEMOKINE LIGAND 2}

Chemokine ligand 2 (CCL2) is a small cytokine that belongs to the CC chemokine family. CCL2 recruits monocytes, memory $\mathrm{T}$ cells, and dendritic cells to the sites of inflammation produced by either tissue injury or infection. ${ }^{[19]}$ The advancing age of renal transplant recipients (RTRs) correlates with the increasing CCL2 concentrations, which 
is reflected in the smoldering inflammation and alterations in MMPs/TIMPs profiles, especially with increased plasma MMP-2 and urine TIMP-1 concentrations. The advanced age of renal transplant recipients has a negative impact on kidney allograft survival through impaired extracellular matrix degradation by the matrix metalloproteinases/tissue inhibitors of metalloproteinases (MMPs/TIMPs) system. ${ }^{[20]}$

\section{CYSTATIN C}

Urinary cystatin $C$ is mainly used as a biomarker of kidney function. The use of cystatin $\mathrm{C}$ alone, or in combination with creatinine, strengthens the association between eGFR and the risk of death and end-stage renal disease across diverse populations. ${ }^{[21]}$ The renal transplant recipients and most of the cystatin C-based equations showed improvements in 30\% and 50\% accuracy compared with the creatinine-based MDRD equation. The cystatin C-based equations may offer an advantage over the MDRD equation in kidney transplant recipients. ${ }^{[2]}$ The elevated levels of urinary CYSC are associated with interstitial fibrosis and tubular atrophy in renal transplant recipients, and may become a useful tool for monitoring kidney allografts. ${ }^{[23]}$

\section{T-CELL IMMUNOGLOBULIN DOMAIN}

The T-cell immunoglobulin domain (TIM3), an immune checkpoint, is a Th1-specific cell surface protein that regulates macrophage activation and enhances the severity of experimental autoimmune encephalomyelitis (EAE) in mice. Tim-3, a novel member of the Tim family of molecules, is the first molecule identified to be specifically expressed on terminally differentiated CD4+ Th1 but not on Th2 cells. ${ }^{[2]}$ Galectin-9, a physiological ligand of Tim3 , bounding to Tim-3 on Th1 and Th17 cells, induces cell apoptosis and/or suppresses cell differentiation. And the activation of the Tim-3-Galectin-9 pathway attenuates cytotoxicity and prolongs the survival of organ allografts in mice. ${ }^{[24]}$

\section{MATRIX METALLOPROTEINASE-7}

Matrilysin, also known as matrix metalloproteinase-7 (MMP-7), is an enzyme in humans that is encoded by the MMP7 gene. Levels of circulating MMPs and TIMPs fluctuate in SLE, and increased MMP-2, MMP-3, MMP7, TIMP-1 and TIMP-2 probably reflect an aggravated

\section{Table 1: Studies of urine biomarkers for diagnosis of established AKI after renal allograft}

Biomarker

L-FABP

CXCL9/CXCL10

[TIMP-2 ]* [IGFBP7]

CCL2

Cystatin C

Tim-3

MMP-7

\section{Comments}

Urinary L-FABP level reflected renal functional deterioration referencing to inulinprognosis for the early detection and clearance; an advance-warning reporter of diseases ${ }^{[27]}$ AKI when used in point-of-care at bedside.

Urinary CXCL9 promising for clinical decision-making following kidney transplantation

Combination of urinary TIMP-2 and urinary IGFBP7 may be important for clinical adjudication of $\mathrm{AKI}$ and risk of kidney failure

Levels of CCL2 at 1 month after transplantation were associated with moderate interstitial fibrosis and tubular atrophy at 6 months on protocol biopsy.

Combination of graft damage biomarkers outperformed serum creatinine in the early after renal allograft based on Le Bricon diagnosis of delayed graft function, and theequation ${ }^{[22]}$

best performance was achieved by a triplemarker approach, using serum creatinine, malondialdehyde and Cystatin C.

Allograft survival in recipients treated with anti-Tim-3 monoclonal antibody or anti-

Gal-9 mAb was significantly shorter than that in control recipients. ${ }^{[35]}$

Urinary MMP7:Cr improves the overall diagnostic performance of urinary CXCL10:Cr for distinguishing normal histology from subclinical and clinical inflammation/injury, but not subclinical inflammation alone.
Noninvasive biomarkers to screen for subclinical tubulitis ${ }^{[29]}$

Identified patients at risk for imminent AKI based on levels of urinary [TIMP-2] * [IGFBP7] ${ }^{[18]}$

Noninvasive identification of patients with or at risk for interstitial fibrosis and inflammation

The most accurate biomarker for AKI

TIM-3 rises as a biomarker for diagnosis acute cellular rejection. ${ }^{[36]}$

MMP7 levels in collagen remodeling reflect acute rejection; the detection of corresponding proteolytic degradation products in urine provides a noninvasive diagnostic approach. ${ }^{[37]}$

\section{Reference \\ Yao $\mathrm{Xu}^{[28]}$; Michael R. Bennett $^{[13]}$}

Hricik DE ${ }^{[14]}$; Rabant $\mathrm{M}^{[30]}$; Paola Romagnani ${ }^{[31]}$

Kathleen D. Liu' ${ }^{[32]}$; Melanie Meersch ${ }^{[17]}$

Ho, Julie [33]; Mazanowska O. ${ }^{[20]}$

Fonseca Isabel ${ }^{[34]}$

Yuanxing $\mathrm{Liu}^{[24]}$

Ho $\mathrm{J}^{[33]}$;

Ling $X B^{[37]}$ 
inflammatory process, whereas lower concentrations of MMP-9 can result from the accumulation of MMPs in the inflamed blood vessels and tissues. ${ }^{[25]}$ Three eighth of urinary MMPs/TIMPs were significantly higher during subclinical tubulitis. Extracellular matrix remodeling is most active during acute tubulo-interstitial inflammation. ${ }^{[2]}$

\section{PROSPECTS AND FUTURE DIRECTIONS}

Early detection of renal damage is of utmost clinical relevance to prevent acute renal failure after kidney transplantation ${ }^{[38]}$ However, the standard clinical tests for detection of kidney injury are insensitive and could detect only advanced stages of injury. It is inspiring that a variety of urinary biomarkers is currently under evaluation to identify biomarkers for early detection of kidney injury. ${ }^{[39]}$ In addition, several urinary biomarkers may help to recognize impaired renal function at an earlier stage, to identify the mechanisms involved in AKI and to improve prognostication. The value of the assays has been validated as a means of reducing the need for kidney transplant biopsy and applying biopsy in a more targeted manner. ${ }^{[40]}$ Together, the previous findings have provided solid foundation for future work aimed at improving kidney transplant outcomes through biomarker-guided decisionmaking. ${ }^{[41]}$ The development of novel biomarkers and detection technologies may lead to the creation of multiplex assays, which allows for the measurement of multiple specific biomarkers simultaneously in the same analyte. Such assays may prove useful for determining the nature of renal injury or the stage of disease in patients. ${ }^{[42]}$ Novel multiplex technologies such as Biomarker Panel Array (BPA) may provide a new perspective for diagnosis and prognosis of renal rejection after kidney transplantation; more importantly, these technologies could significantly improve the sensitivity and specificity in reflecting the clinical manifestations. It is important to note that the advancement of proteomics technologies may become a critical drive for the discovery of novel urinary biomarkers which could specifically and accurately reflect underlying molecular events of allograft rejection. The validation and assessment of the performance of urinary biomarkers for allograft rejection remains a significant, costly, and high-risk undertaking process. ${ }^{[2]}$

\section{Conflict of Interest}

None declared.

\section{REFERENCES}

1. Greenan G, Ahmad SB, Anders MG, Leeser A, Bromberg JS, Niederhaus SV. Recreational Marijuana Use is Not Associated with Worse Outcomes
After Renal Transplantation. Clin Transplant 2016; Aug 4. [Epub ahead of print]

2. Blok JJ, Braat AE, Adam R, Burroughs AK, Putter H, Kooreman NG, et al. Validation of the donor risk index in orthotopic liver transplantation within the Eurotransplant region. Liver Transpl 2012; 18: 112-9.

3. Lentine KL, Axelrod D, Klein C, Simpkins C, Xiao H, Schnitzler MA, et al. Early clinical complications after $\mathrm{ABO}$-incompatible live-donor kidney transplantation: a national study of Medicare-insured recipients. Transplantation 2014; 98: 54-65.

4. Hinchliffe TE, Lin ZT, Wu T. Protein arrays for biomarker discovery in lupus. Proteomics Clin Appl 2016; 10: 625-34.

5. Sellarés J, de Freitas DG, Mengel M, Reeve J, Einecke G, Sis B, et al. Understanding the causes of kidney transplant failure: the dominant role of antibody-mediated rejection and nonadherence. Am J Transplant 2012; 12: 388-99.

6. Krüger B, Krick S, Dhillon N, Lerner SM, Ames S, Bromberg JS, et al. Donor Toll-like receptor 4 contributes to ischemia and reperfusion injury following human kidney transplantation. Proc Natl Acad Sci USA 2009; 106: 3390-5.

7. Naesens M, Kuypers DR, De Vusser K, Evenepoel P, Claes K, Bammens $\mathrm{B}$, et al. The histology of kidney transplant failure: a long-term follow-up study. Transplantation 2014; 98: 427-35.

8. Mayeux R. Biomarkers: potential uses and limitations. NeuroRx 2004; 1: $182-8$.

9. Mikolasevic I, Racki S, Lukenda V, Pavletic-Persic M, Milic S, Orlic L. Non-alcoholic fatty liver disease; a part of the metabolic syndrome in the renal transplant recipient and possible cause of an allograft dysfunction. Med Hypotheses 2014; 82: 36-9.

10. Wu T, Du Y, Han J, Singh S, Xie C, Guo Y, et al. Urinary angiostatin--a novel putative marker of renal pathology chronicity in lupus nephritis. Mol Cell Proteomics 2013; 12: 1170-9.

11. Coca SG, Parikh CR. Urinary biomarkers for acute kidney injury: perspectives on translation. Clin J Am Soc Nephrol 2008; 3: 481-90.

12. Kim SC, Page EK, Knechtle SJ. Urine proteomics in kidney transplantation. Transplant Rev (Orlando) 2014; 28: 15-20.

13. Bennett MR, Nehus E, Haffner C, Ma Q, Devarajan P. Pediatric reference ranges for acute kidney injury biomarkers. Pediatr Nephrol 2015; 30: 677-85.

14. Hricik DE, Nickerson P, Formica RN, Poggio ED, Rush D, Newell KA, et al. Multicenter validation of urinary CXCL9 as a risk-stratifying biomarker for kidney transplant injury. Am J Transplant 2013; 13: 2634-44.

15. Fenwick PS, Macedo P, Kilty IC, Barnes PJ, Donnelly LE. Effect of JAK Inhibitors on Release of CXCL9, CXCL10 and CXCL11 from Human Airway Epithelial Cells. PLoS One 2015; 10: e0128757.

16. Corbera-Bellalta M, Planas-Rigol E, Lozano E, Terrades-García N, Alba MA, Prieto-González S, et al. Blocking interferon $\gamma$ reduces expression of chemokines CXCL9, CXCL10 and CXCL11 and decreases macrophage infiltration in ex vivo cultured arteries from patients with giant cell arteritis. Ann Rheum Dis 2016; 75: 1177-86.

17. Meersch M, Schmidt C, Van Aken H, Martens S, Rossaint J, Singbartl K, et al. Urinary TIMP-2 and IGFBP7 as early biomarkers of acute kidney injury and renal recovery following cardiac surgery. PLoS One 2014; 9 : e93460.

18. Bihorac A, Chawla LS, Shaw AD, Al-Khafaji A, Davison DL, Demuth GE, et al. Validation of cell-cycle arrest biomarkers for acute kidney injury using clinical adjudication. Am J Respir Crit Care Med 2014; 189: 932-9.

19. Kitamura T, Qian BZ, Soong D, Cassetta L, Noy R, Sugano G, et al. CCL2-induced chemokine cascade promotes breast cancer metastasis by enhancing retention of metastasis-associated macrophages. J Exp Med 2015; 212: 1043-59.

20. Mazanowska O, Zabińska, Kościelska-Kasprzak, Kamińska D, Banasik M, Krajewska M, et al. Advanced age of renal transplant recipients correlates with increased plasma concentrations of interleukin-6, chemokine ligand 2 (CCL2), and matrix metalloproteinase 2 , and urine concentrations of 
CCL2 and tissue inhibitor of metalloproteinase 1. Transplant Proc 2014; 46: 2640-3.

21. Shlipak MG, Matsushita K, Ärnlöv J, Inker LA, Katz R, Polkinghorne KR, et al. Cystatin $\mathrm{C}$ versus creatinine in determining risk based on kidney function. N Engl J Med 2013; 369: 932-43.

22. Harman G, Akbari A, Hiremath S, White CA, Ramsay T, Kokolo MB, et al. Accuracy of cystatin C-based estimates of glomerular filtration rate in kidney transplant recipients: a systematic review. Nephrol Dial Transplant 2013; 28: 741-57.

23. Mendes MF, Salgado JV, de Ribamar Lima J, Ferreira TC, Silva GE, Filho NS. Increased urinary cystatin C level is associated with interstitial fibrosis and tubular atrophy in kidney allograft recipients. Clin Biochem 2015; 48: 546-9.

24. Qiao X, Jiang K, Nie J, Fan K, Zheng Z, Wang J, et al. Increased expression of Tim- 3 and its ligand Galectin-9 in rat allografts during acute rejection episodes. Biochem Biophys Res Commun 2014; 445: 542-8.

25. Afkarian M, Zelnick LR, Ruzinski J, Kestenbaum B, Himmelfarb J, de Boer $\mathrm{IH}$, et al. Urine matrix metalloproteinase-7 and risk of kidney disease progression and mortality in type 2 diabetes. J Diabetes Complications 2015; 29: 1024-31.

26. Hirt-Minkowski P, Marti HP, Hönger G, Grandgirard D, Leib SL, Amico $\mathrm{P}$, et al. Correlation of serum and urinary matrix metalloproteases/tissue inhibitors of metalloproteases with subclinical allograft fibrosis in renal transplantation. Transpl Immunol 2014; 30: 1-6.

27. Negishi K, Noiri E, Doi K, Maeda-Mamiya R, Sugaya T, Portilla D, et al. Monitoring of urinary L-type fatty acid-binding protein predicts histological severity of acute kidney injury. Am J Pathol 2009; 174: 1154-9.

28. Xu Y, Xie Y, Shao X, Ni Z, Mou S. L-FABP: A novel biomarker of kidney disease. Clin Chim Acta 2015; 445: 85-90.

29. Schaub S, Nickerson P, Rush D, Mayr M, Hess C, Golian M, et al. Urinary CXCL9 and CXCL10 levels correlate with the extent of subclinical tubulitis. Am J Transplant 2009; 9: 1347-53.

30. Rabant M, Amrouche L, Morin L, Bonifay R, Lebreton X, Aouni L, et al. Early Low Urinary CXCL9 and CXCL10 Might Predict Immunological Quiescence in Clinically and Histologically Stable Kidney Recipients. Am J Transplant 2016; 16: 1868-81.

31. Romagnani P, Crescioli C. CXCL10: a candidate biomarker in transplantation. Clin Chim Acta 2012; 413: 1364-73.

32. Liu KD, Vijayan A, Rosner MH, Shi J, Chawla LS, Kellum JA. Clinical adjudication in acute kidney injury studies: findings from the pivotal
TIMP-2*IGFBP7 biomarker study. Nephrol Dial Transplant 2016; Jun 23. [Epub ahead of print]

33. Ho J, Wiebe C, Gibson IW, Hombach-Klonisch S, Gao A, Rigatto $\mathrm{C}$, et al. Elevated urinary CCL2: $\mathrm{Cr}$ at 6 months is associated with renal allograft interstitial fibrosis and inflammation at 24 months. Transplantation 2014; 98: 39-46.

34. Fonseca I, Reguengo H, Oliveira JC, Martins LS, Malheiro J, Almeida $\mathrm{M}$, et al. A triple-biomarker approach for the detection of delayed graft function after kidney transplantation using serum creatinine, cystatin C, and malondialdehyde. Clin Biochem 2015; 48: 1033-8.

35. Liu Y, Ji H, Zhang Y, Shen X, Gao F, He X, et al. Recipient T cell TIM-3 and hepatocyte galectin- 9 signalling protects mouse liver transplants against ischemia-reperfusion injury. J Hepatol 2015; 62: 563-72.

36. Naka EL, Ponciano VC, Cenedeze MA, Pacheco-Silva A, Câmara NO. Detection of the Tim-3 ligand, galectin-9, inside the allograft during a rejection episode. Int Immunopharmacol 2009; 9: 658-62.

37. Ling XB, Sigdel TK, Lau K, Ying L, Lau I, Schilling J, et al. Integrative urinary peptidomics in renal transplantation identifies biomarkers for acute rejection. J Am Soc Nephrol 2010; 21: 646-53.

38. Singh S, Wu T, Xie C, Vanarsa K, Han J, Mahajan T, et al. Urine VCAM-1 as a marker of renal pathology activity index in lupus nephritis. Arthritis Res Ther 2012; 14: R164.

39. Wu T, Fu Y, Brekken D, Yan M, Zhou XJ, Vanarsa K, et al. Urine proteome scans uncover total urinary protease, prostaglandin $\mathrm{D}$ synthase, serum amyloid $\mathrm{P}$, and superoxide dismutase as potential markers of lupus nephritis. J Immunol 2010; 184: 2183-93.

40. Wu T, Ding H, Han J, Arriens C, Wei C, Han W, et al. AntibodyArray-Based Proteomic Screening of Serum Markers in Systemic Lupus Erythematosus: A Discovery Study. J Proteome Res 2016; 15: 2102-14.

41. Wu T, Xie C, Wang HW, Zhou XJ, Schwartz N, Calixto S, et al. Elevated urinary VCAM-1, P-selectin, soluble TNF receptor-1, and CXC chemokine ligand 16 in multiple murine lupus strains and human lupus nephritis. J Immunol 2007; 179: 7166-75.

42. Tesch GH. Review: Serum and urine biomarkers of kidney disease: A pathophysiological perspective. Nephrology (Carlton) 2010; 15: 609-16.

How to cite this article: Wang $\mathrm{H}$, Lin Z, Yuan $\mathrm{Y}$, Wu T. Urine biomarkers in renal allograft. J Transl Intern Med 2016; 4: 109-13. 